This document is published in:

Polymer Degradation and Stability, vol. 98, issue 12 (2013), pp. 2546-2555

DOI: http://dx.doi.org/10.1016/j.polymdegradstab.2013.09.015

(C) 2013 Elsevier Ltd. 


\title{
Effect of the particle size and solids volume fraction on the thermal degradation behaviour of Invar 36 feedstocks
}

\author{
J. Hidalgo a,*, J.P. Fernández-Blázquez ${ }^{c}$, A. Jiménez-Morales ${ }^{a}$, T. Barriere ${ }^{\mathrm{b}}$, J.C. Gelin ${ }^{\mathrm{b}}$, \\ J.M. Torralba ${ }^{\mathrm{a}, \mathrm{c}}$ \\ ${ }^{a}$ Materials Science Department at Carlos III University of Madrid, Avd. Universidad 30, Leganés 28911, Spain \\ ${ }^{\mathrm{b}}$ Applied Mechanics Department at FEMTO-ST Institute, Besançon, France \\ ${ }^{\mathrm{c}}$ IMDEA Materials Institute, Getafe, Spain
}

\section{A R T I C L E I N F O}

Keywords:

Powder injection moulding

Invar 36

Cellulose acetate butyrate

Polyethylene glycol

Thermal degradation

\begin{abstract}
A B S T R A C T
Degradation kinetics and the thermal stability of Invar 36 powder injection moulding feedstocks (PIM) based on cellulose acetate butyrate (CAB) and polyethylene glycol (PEG) binders were investigated using simultaneous thermogravimetric analysis (STA) and differential scanning calorimetry (DSC). The initial decomposition temperature (IDT) and the integral procedure decomposition temperature (IPDT) were used to analyse the thermal stability of the binder system as a function of the solid loading content and powder particle size. The degradation kinetics was studied, and the process apparent activation energies were assessed using isoconversional methods. All the methodologies revealed changes in the thermal degradation behaviours of the feedstocks for solid loadings that were previously determined to corre spond to optimal solid loadings using other experimental procedures. The studies also contrast previous similar findings with a ceramic powder. Therefore these results strengthen the proposal of thermody namic degradation studies of feedstocks as an alternative or complementary technique to determine optimal solid loading contents in metal injection moulding (MIM).
\end{abstract}

\section{Introduction}

Powder injection moulding (PIM) is one of the main manufacturing processes used to produce small parts with complex geometries, thin walls and in large production batches [1]. This technology is applied to a wide variety of ceramics (commonly referred as ceramic injection moulding, CIM), metals (also referred as metal injection moulding, MIM) and combinations thereof [2]. The process consists of mixing a fine powder material with different polymer like compounds, the so called binder system. The binder system provides sufficient fluidity to the newly created feedstock to allow mould filling during injection. However, it will be removed during the subsequent debinding process, thus it has to allow an easy extraction. The binder system must be added in such quantities so as to optimise the process conditions and time required.

Elimination of the binder system out of the green body is the bottle neck in MIM processes and a critical and delicate step. It requires tailored optimisation of the time-temperature cycle in

\footnotetext{
* Corresponding author. Tel.: +34 916249482.

E-mail address: jhidalgo@ing.uc3m.es (J. Hidalgo).
}

order to be economical and effective while avoiding faults like undesirable blistering [3]. For multi component binders obtaining optimal thermal cycles is a difficult task that conventionally has been performed by "trial and error". However recent works pro pose to investigate the binder decomposition kinetics in order to identify points of rapid weight loss to avoid green parts damage, to speed up the debinding process and lower the overall cost [4-6].

The thermal burn out of organic components during debinding processes of MIM feedstock is intricate. There are several decom position mechanism involved which are also dependant on several factors. Transport and elementary reactions steps could be rate limiting during debinding. Transport mechanisms would become more influential with increasing sample thickness, whereas in thin specimens such as in micro pieces, polymer decomposition kinetics would play a predominant role [7].

In previous works [8] we have proposed thermogravimetric analysis as an alternative to determine optimal solid loading for ceramic feedstocks while studying their thermal stability and decomposition kinetics. In this work same methods are contrasted with metallic powders. Calorimetric and thermogravimetric ana lyses were performed to study the thermal decomposition of an Invar 36 alloy $\left(\mathrm{Fe}_{0.64} \mathrm{Ni}_{0.36}\right)$ feedstocks composed of cellulose ace tate butyrate $(\mathrm{CAB})$ and polyethylene glycol (PEG) binders in an 
inert nitrogen atmosphere. Binder and feedstock temperature sta bility and decomposition kinetics were investigated using integral procedure decomposition temperature (IPDT) and isoconversional methodologies, respectively. A number of isoconversional methods were used, and their convenience discussed.

Several Invar 36 feedstocks consisting of mixtures with different proportions of constituents were investigated. The volume frac tions of metallic powders as well as the powder particle sizes were varied in this study. These variations allowed the effect of the effective powder surface on the interactions of CAB and PEG mol ecules with Invar 36 particles and the channel volume used to remove volatiles to be elucidated. The degradation of the binder system was studied to evaluate the extent of the influence that particles exert on the decomposition of $C A B$ and PEG. The results reveal changes in the thermal degradation behaviour with solid loading and particle size. Based on these findings, we have con trasted the suitability to determine the optimum solid loading conditions using thermogravimetric tools.

\section{Thermal stability studies}

The initial decomposition temperature (IDT) and the integral procedure decomposition temperature (IPDT) were used to analyse the thermal stability of the binder system as a function of the solid loading content and particle size. The IDT indicates the apparent thermal stability of binder system with regard to the maximum feedstock processing rate and moulding temperatures. The IDT is determined by the onset weight loss in the TGA curves of the different feedstocks. In this work IDT corresponds to the tempera ture at which the $2 \%$ in mass of the organic compounds was lost. The IPDT determined from the residual weight fraction in the TGA is a reproducible datum that can be consistently determined for diverse materials and represents both a truly comprehensive index of intrinsic thermal stability and a real temperature that has prac tical significance. The method for calculating the IPDT is proposed by Doyle $[9,10]$ and converts the area under the TGA curve into a value that approximately represents the characteristic end of volatilisation temperature, $T_{\mathrm{a}^{*}}$ (Eq. (1)):

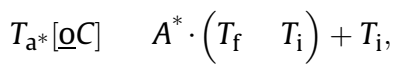

where $A^{*}$ is the area under the TGA curve within the evaluated temperature range (defined by initial temperature $T_{\mathrm{i}}$ and the final temperature $T_{\mathrm{f}}$ ) and normalised with respect to both the residual weight and temperature. However, the IPDT takes into account the amount of refractory or non volatile residues at $T_{\mathrm{f}}$ by means of the coefficient $\mathrm{K}^{*}$. Therefore, the method to calculate the IPDT is described in Eq. (2):

$$
\text { IPDT }[\underline{o} C] \quad A^{*} \cdot K^{*} \cdot\left(\begin{array}{ll}
T_{\mathrm{f}} & T_{\mathrm{i}}
\end{array}\right)+T_{\mathrm{i}} .
$$

A representation of the areas $S_{1}, S_{2}$ and $S_{3}$ used to calculate $A^{*}\left[A^{*} \quad\left(S_{1}+S_{2}\right) /\left(S_{1}+S_{2}+S_{3}\right)\right]$ and $K^{*}\left[K^{*} \quad\left(S_{1}+S_{2}\right) / S_{1}\right]$ is shown in Fig. 1. In these cases, the IPDT represents the inherent thermal stability of the binder system and describes the entire binder degradation process in terms of the decomposition and vapor isation of the different volatiles taking into account differences in the solid loading percentages. The higher the IPDT is, the more thermally stable the feedstock is. Thus, the binder components will resist higher temperatures until complete volatilisation occurs.

From the IPDT definition, it is clear that this value will be influenced by the selection of initial and final temperatures. To the best of the authors knowledge there is not a standard that defines where to set these temperatures. Hence, there is a limitation of using IPDT value when different systems are compared. In this

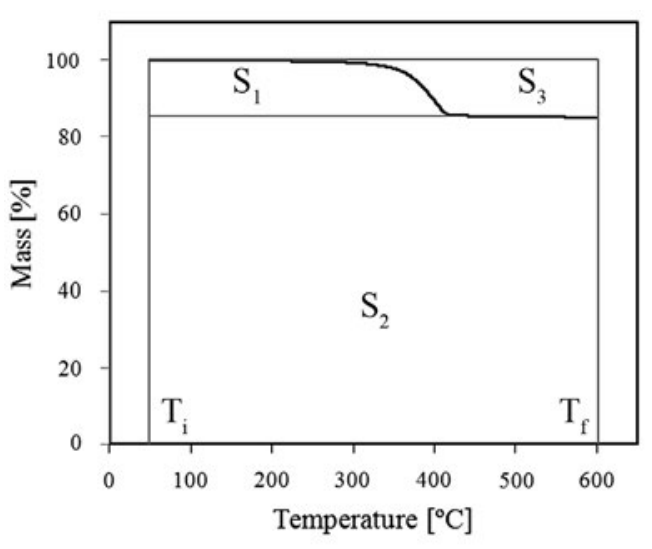

Fig. 1. Scheme of the areas $S 1, S 2$ and $S 3$ for the IPDT assessment.

work initial temperature was selected approximately considering the $20 \%$ below the IDT, which resulted to be $50{ }^{\circ} \mathrm{C}$. For the final temperature, approximately the $20 \%$ above the end of degradation temperature was selected, which resulted to be $600{ }^{\circ} \mathrm{C}$. For com parison purposes this limits were maintained when determining the IPDT of different feedstocks and binder system components.

\section{Degradation kinetic studies}

A thorough discussion about the convenience of using model free isoconversional methods to analyse thermal decomposition of MIM feedstocks could be found in the work of Salehi et al. [7]. Model free methods turn out to be the most reliable way to determine kinetic parameters of thermally activated complex processes, because the prior knowledge of the reaction mechanism is not necessary [11]. These methods are based on studying the degree of conversion with respect to temperature at different heating rates. The degree of conversion for a certain temperature $\alpha$ is defined as the ratio of the actual weight loss $m(T)$ to the total weight loss, as expressed in Eq. (3):

$\alpha(T) \quad \frac{m_{0} m(T)}{m_{0} \quad m_{\infty}}$.

The variables $m_{0}$ and $m_{\infty}$ correspond to the initial and final masses, respectively. In the model free methods apparent activa tion energy is calculated directly from the TGA curves. The term apparent activation energy comes for the uncertainty of the phys ical process associated to this activation energy. Activation energy most likely takes into account several different processes occurring at the same time. The rate of degradation, $\mathrm{d} \alpha / \mathrm{d} t$, is assumed to depend on the temperature and weight of the sample. Therefore, the main advantage of eliminating the necessity of a kinetic model is clouded by the influence of the sample mass and size in the apparent kinetics parameters calculated. Furthermore these pa rameters could be influenced by the thermal lag across the spec imen [12]. To reduce possible errors regarding size and mass, samples of similar dimensions and masses were employed. It is assumed for the thin disks employed that the binder removal rate is predominantly determined by decomposition rates of organics and not by transport processes.

In literature a wide variety of non isothermal models could be found. The lack of agreement among kinetic parameters calculated from the same set of experimental data using different methods of mathematical analysis is disturbing. It has been shown that the causes for these differences are due to the intrinsic nature of the methods. In this work Flynn-Wall-Ozawa (FWO) and Kissinger- 
Akahira-Sunose (KAS) integral methods are selected to estimate apparent activation energy values. They are based in the integral of the following expression (Eq. (4)):

$\frac{\mathrm{d} \alpha}{f(\alpha)} \quad\left(\frac{k_{0} \cdot \exp \left(\quad E_{\mathrm{a}} / R \cdot T\right)}{\beta}\right) \cdot \mathrm{d} T$,

where $k_{0}$ represents the pre exponential factor, or frequency factor; $E_{\mathrm{a}}$ is the apparent activation energy; $R$ is the gas constant; $T$ is the absolute temperature; and $\beta$ is the heating rate $(\mathrm{d} T / \mathrm{d} t)$.

The FWO $[13,14]$ method is based on the principle that a reaction rate at a constant conversion is only a function of temperature. Using Doyle's approximation [15] of the temperature integral $p\left(E_{\mathrm{a}}\right)$ $R \cdot T$ ) and applying logarithms, the following expression is produced (Eq. (5)):

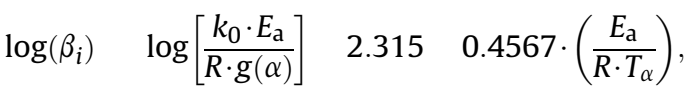

where $g(\alpha)$ represents the integral of $\mathrm{d} \alpha / f(\alpha)$. Considering a con stant conversion, the representation of $\log \beta$ vs. $1 / T$ should be a straight line with a slope defined as $\mathrm{d}(\log \beta) / \mathrm{d}(1 / T) \quad 0.4567\left(E_{\mathrm{a}} /\right.$ $R \cdot T)$. Therefore, the activation energy may be calculated from Eq. (6) as follows:

$E_{\mathrm{a}} \quad$ slope $\cdot \frac{R}{0.457}$.

The KAS method [16,17] (Eq. (7)) is based on the Coats-Redfern [18] approximation of the temperature integral $p\left(E_{\mathrm{a}} / R \cdot T\right)$ and considering that $2 R \cdot T / E_{\mathrm{a}}$ is much less than unity. The resulting relationships are expressed as:

$\ln \left(\frac{\beta_{i}}{T_{\alpha}^{2}}\right) \quad \ln \left(\frac{k_{0} \cdot R}{E_{\mathrm{a}} \cdot g(\alpha)}\right) \quad \frac{E_{\mathrm{a}}}{R \cdot T_{\alpha}}$.

Thus, the plot of $\ln \left(\beta / T^{2}\right)$ vs. $1 / T^{2}$ for constant values of $\alpha$ and $\alpha i$ should be a straight line with a slope that can be used to calculate the apparent activation energy (Eq. (8)):

$$
E_{\mathrm{a}} \quad \text { slope } R \text {. }
$$

Some of the main shortcomings of both methods are using an approximation of the temperature integral and the selection of the integral limits, but the validity of the calculated apparent activation energies is assumed good enough for the general proposes of this work.

\section{Experimental procedure}

\subsection{Materials}

Gas atomised spherical Invar 36 powders were supplied by Sandvik Osprey Ltd., (UK). Two powders with different particle sizes were selected in this study. The coarsest powder was denoted as INV1 whereas the finest powder was denoted as INV2. A Malvern 2000 laser scattering device was used to measure the particle size distribution parameters. The powders specific surface areas were measured with a Micrometrics Gemini VII BET measurement de vice. All the particles parameters for both INV1 and INV2 are collected in Table 1.

A binder system based on PEG and CAB was selected. These binders have been demonstrated to be effective for PIM and provide improved properties when compared to other binder systems $[19,20]$. However, there are no published comprehensive studies on their thermal degradation when Invar 36 particles are added. The binders consist of two types of CAB: CAB381 0.1 and CAB551 0.01
Table 1

Particles parameters for INV and INV2.

\begin{tabular}{lllllll}
\hline & \multicolumn{2}{l}{ Particle size distribution } & & \multicolumn{2}{l}{ Specific area } \\
\cline { 2 - 4 } & $d_{10}[\mu \mathrm{m}]$ & $d_{50}[\mu \mathrm{m}]$ & $d_{90}[\mu \mathrm{m}]$ & $S_{\mathrm{w}}$ & & BET $\left[\mathrm{m}^{2} / \mathrm{g}\right]$ \\
\hline INV1 & 5.0 & 7.7 & 11.4 & 7.2 & 0.14 \\
INV2 & 1.9 & 3.4 & 5.9 & 8.3 & 0.24 \\
\hline
\end{tabular}

(Eastman). Each of these binder components has different per centages of butyryl, acetyl and hydroxyl groups. They were blended with two types of PEG (Sigma Aldrich) with different average mo lecular weights. The composition of the binder system and the characteristics of the components are shown in Table 2.

\subsection{Preparation of the samples}

A Rheomix 600 Haake rheometer coupled with a Haake Rheo cord 252p module was used for the mixing all feedstock compo nents. Different feedstock compositions, ranging from 57.5 vol.\% (minimum solid loading tested) to 72.5 vol.\% (maximum solid loading tested), were investigated in 2.5 vol.\% increments. A tem perature of $150{ }^{\circ} \mathrm{C}$ was used for all the batches, and the mixing chamber was filled with feedstock to $72 \%$ of the total volume (the chamber volume is $69 \mathrm{~cm}^{3}$ ). A rotor speed of $50 \mathrm{rpm}$ was employed to mix the feedstock and the binder for $60 \mathrm{~min}$ to ensure complete homogenisation. Thin samples of around the same size and weight were prepared by warm pressing of the as prepared feedstocks to perform thermogravimetric and calorimetric analysis.

\subsection{Termogravimetric and calorimetric analyses (STA and DSC)}

A Perkin Elmer STA 6000 device was used to thermodynamically evaluate the decomposition of the binder and the feedstock. This device allows the simultaneous acquisition of the thermogravi metric TGA curves and the DTA/DSC curves. The examined tem perature range was from $50{ }^{\circ} \mathrm{C}$ to $650{ }^{\circ} \mathrm{C}$. Calcium oxalate was employed for thermogravimetric calibration, whereas elemental indium (99.999\% pure) was used for heat and temperature cali bration. An inert nitrogen atmosphere was employed with a flow rate of $40 \mathrm{ml} / \mathrm{min}$. Feedstock pellets of approximately the same morphology and weight were used for the analysis. The net weight of the binder in the feedstock was constant for all experiments, with a minimum of $5 \mathrm{mg}$ for all cases. An open aluminium oxide crucible was used to perform the experiments. For a thorough evaluation of the decomposition kinetics, four heating rates were evaluated: $10{ }^{\circ} \mathrm{C} / \mathrm{min}, 15^{\circ} \mathrm{C} / \mathrm{min}, 20^{\circ} \mathrm{C} / \mathrm{min}$ and $25^{\circ} \mathrm{C} / \mathrm{min}$. The buoyancy effect in TGA has been taken into account by carrying out empty crucible runs and subtracting the resulting weight differ ences from the subsequent sample mass loss data [21].

A TA Instruments Q200 differential scanning calorimeter (DSC) equipped with a refrigerated cooling system was also employed to contrast STA results. The base line was calibrated in scanning the temperature domain with an empty pan. The temperature and the enthalpy were calibrated using indium as a standard. All the

Table 2

Binder composition.

\begin{tabular}{lllll}
\hline Component & Acetyl $^{\text {a }}$ & Butyryl $^{\text {a }}$ & Hydroxyl $^{\text {a }}$ & \%vol \\
\hline CAB381-0.1 & 13 & 37 & 1.5 & 30 \\
CAB551-0.01 & 2 & 53 & 1.5 & 10 \\
PEG20k & & & & 58 \\
PEG10k & & & & 2 \\
\hline
\end{tabular}

a Percentage of side groups in chain. 
experiments were carried out with an aluminium pan with 8$12 \mathrm{mg}$ of sample. These experiments allow data acquisition at lower temperatures ranges with a better resolution than with combined STA technique. Typical heating-cooling-heating cycles were pro grammed to delete the thermal history of the feedstocks and to evaluate the melting and crystallisation temperatures. The experi mental temperature range varies from $20-190{ }^{\circ} \mathrm{C}$ to avoid any possible degradation of the components and to allow complete crystallisation. Heating rates of $10^{\circ} \mathrm{C} / \mathrm{min}$ were selected.

\subsection{Polarised light microscopy}

Investigation of PEG crystallization in different systems was performed by an Olympus GX71 light microscope with light polarising filters. A thin layer on around $0.5 \mathrm{~mm}$ was created by facing two microscope slides and pressing the sample in its melting state. Samples were melted at $160{ }^{\circ} \mathrm{C}$ during $30 \mathrm{~min}$. Then they were air quenched at same cooling rates for comparison purposes.

\section{Results and discussion}

\subsection{DSC investigations}

Characteristic melting and crystallisation PEG peaks as well as CAB transition temperatures were investigated by DSC measure ments. This study was done for the single binder components, the binder and the different feedstocks. Changes in the peaks features in terms of temperature displacements and specifics enthalpies increment may reveal the occurrence of interactions between binder components and between binder components and powder particles. These interactions may affect the decomposition behav iour of the feedstocks. Salehi et al. [7] demonstrated that the kneading effect and the presence of powder particles in PS promote the formation of weak links between polymer chains. This was related with a lowering of the degradation activation energy which enables the weak links to act as initiation points for PS degradation.

In Fig. 2 the DSC curves of the binder system and the binder components are plotted. Table 3 collects characteristic events during the heating and the cooling along the DSC curves. The value for the second heating and cooling cycles is presented. During the first heating cycle a melting peak was observed for CAB381 and CAB551 occurring at $147.7{ }^{\circ} \mathrm{C}$ and $125.5{ }^{\circ} \mathrm{C}$ respectively. No peak appears during subsequent cooling and heating cycles, therefore crystallisation did not take place at the cycle conditions as it occurred with the PEG.

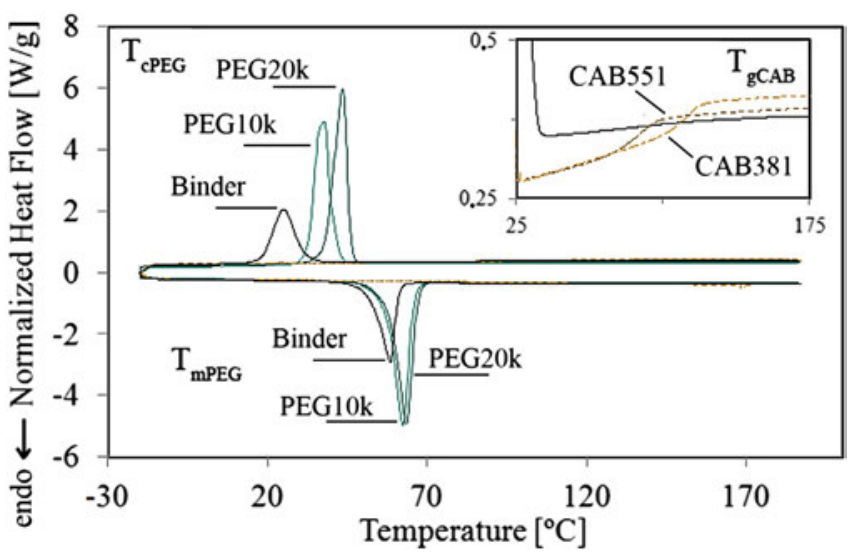

Fig. 2. Normalised heat flow vs. temperature DSC curves for the binder system and the single binder components.
Table 3

Characteristic events during the heating and cooling processes of the single binder components and the binder system. $T_{\text {mPEG }}$ : PEG melting temperatures; $T_{\mathrm{cPEG}}$ : PEG crystallisation temperatures; $T_{\mathrm{gCAB}}$ : glass transition of $\mathrm{CAB} ; \Delta H_{\mathrm{mPEG}}$ : specific enthalpy of melting relative to the PEG mass; $\Delta H_{\mathrm{CPEG}}$ : specific enthalpy of crystallisation relative to the PEG mass.

\begin{tabular}{lcccll}
\hline & PEG10k & PEG20k & CAB381 & CAB551 & Binder \\
\hline$T_{\text {mPEG }}\left[{ }^{\circ} \mathrm{C}\right]$ & 62.5 & 63.4 & & & 58.5 \\
$\Delta H_{\text {mPEG }}\left[\mathrm{J} / \mathrm{g}_{\mathrm{PEG}}\right]$ & 175.6 & 181.7 & & & 240.6 \\
$T_{\mathrm{CPEG}}\left[{ }^{\circ} \mathrm{C}\right]$ & 37.7 & 43.4 & & & 25.0 \\
$\Delta H_{\mathrm{CPEG}}\left[\mathrm{J} / \mathrm{g}_{\mathrm{PEG}}\right]$ & 172.1 & 177.5 & & & 218.4 \\
$T_{\mathrm{gCAB}}\left[{ }^{\circ} \mathrm{C}\right]$ & & & 104.1 & 89.3 & No detected \\
\hline
\end{tabular}

Comparing different binder components and binder system, differences in the melting and crystallising of PEG10k and PEG20k are comprehensible due to the differences in their molecular weights. Differences in the glass transitions of the $C A B$ are also predictable due to differences in the side groups' composition. The PEG20k and PEG10k melting points are very similar in contrast with the differences observed in their crystallisation peaks. The melting point is less sensitive to variations of the molecular weight but this feature or others affect on the crystallisation kinetics of the PEGs. Actually, differences in the crystallisation behaviour were observed by controlled temperature polarised light microscopy experiments during cooling. The PEG20k began to crystallise at higher temperatures and a higher density of nucleation points was observed. Contrary, the PEG10k began to crystallise at lower tem peratures and less nucleating points were observed. These results are consistent with the DSC measurements. As result big spheru lites are observed for PEG10k compared with those for PEG20k as shown in the POM images presented in Fig. 3.

The melting and crystallization peaks associated to the PEG components in the binder system fell drastically to temperatures far below those of the single components, however, the enthalpy related to PEG mass increased. These facts can be explained by a certain miscibility of CAB and PEG polymer chains, which is sup ported by two observations: a) Both CAB381 and CAB551 presented a glass transition which was not detected in the binder system; b) The increase of melting enthalpy related to PEG mass suggests that CAB takes part in the formation of PEG crystals. It would be explained by a widening of glass transition temperature range for the CABs and a displacement of this glass transition to lower temperatures. This would be agreed with and strengthen the assumption of the interactions between the CABs and PEGs binder components. In addition to DSC measurements, controlled tem perature polarised light microscopy experiments during cooling were carried out. Onset of crystallization of PEG spherulites in the binder system occurred at lower temperatures than those for single PEGs which is coherent with DSC results. However a higher density of nucleation points were observed compared with single PEGs. This also emphasises interactions between components; the CABs molecules most likely act as a nucleation agent but also retard the crystallisation during cooling. A polarised optic microscope (POM) image of the binder system is shown in Fig. 4. A decrease of the spherulites size in comparison with Fig. 3a-b, which represents the single PEGs, could be observed. This image also shows a zone that presents a variation in the spherulite morphology. The spherulites borders are blurred most likely as consequence of high concen tration of $\mathrm{CAB}$ and certain interactions between both substances.

Table 4 shows the influence of the solid loading and particle size on the melting and crystallisation peaks associated with the PEGs. One remarkable finding is the general observation of these peaks at temperatures between those for the PEGs and the binder system. A reasonable explanation of this fact could be given considering in teractions between the binder components and the metallic 


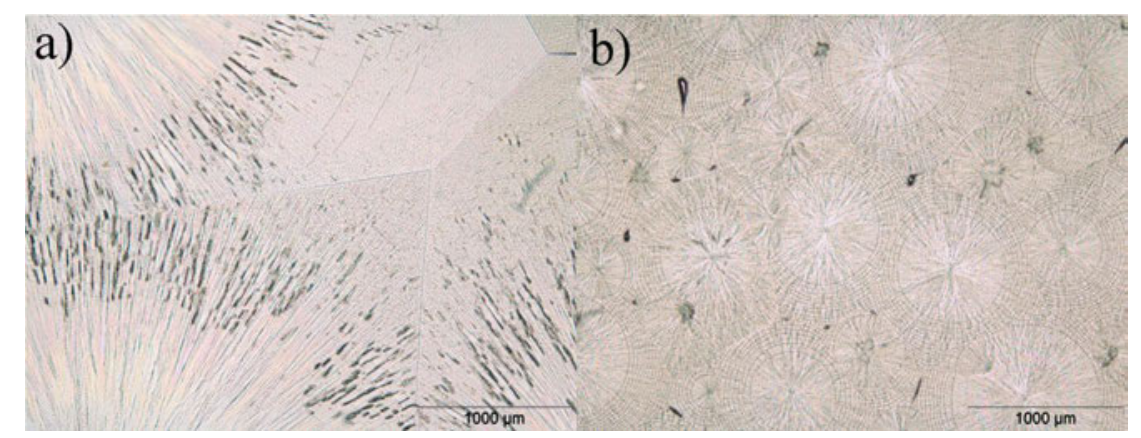

Fig. 3. POM images of PEG spherulites for a) PEG10k and b) PEG20k.

powders. Most probably PEG chains interact with the powder which is evidenced by the feedstocks PEGs' peak displacement to higher temperatures than the PEGs' peak corresponding to the binder system. Invar 36 particle surfaces are covered by a natural occurring oxide layer. Namely, hydroxyl groups are well reported to be present on metal oxide surfaces [22]. Several works studied the interactions between PEG and silica or alumina powders by infrared spectroscopy $[23,24]$. They state that hydrogen bonds are formed between the PEG etheric oxygen group and the metal sur face hydroxyl group. PEGs' chains, if thermally or mechanically driven, may possibly have a higher interaction with oxide surface. In the same way, CABs interactions with metallic powders are not discarded but they could not be proven, neither experimentally nor by literature. Further studies should be carried out to ascertain the nature of possible bondings.

The melting and crystallisation temperatures are not very influenced by the solid loading up to certain points that depend on the particle size. In INV1 feedstock a pronounced melting and crystallisation temperature fall occurs at 70 vol.\% solid loading whereas the similar behaviour is observed for INV2 at 65 vol.\% solid loadings. These compositions were associated with critical powder volume content (CPVC) situations in previous studies. The enthalpy values also show similar behaviours but the drop on the enthalpy values occurred at $60 \mathrm{vol} \%$ in the case of INV2. As long as there is enough inter particle gap to allow the formation of PEG's crystal line regions, the phase transitions will not be critically altered. In such cases the effect of high free volume for crystallisation prevails

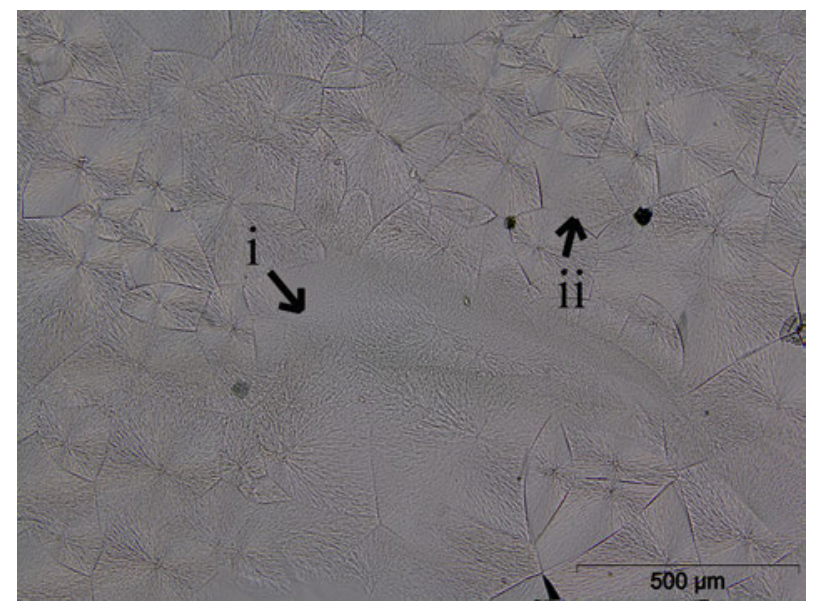

Fig. 4. POM image of the binder system: i) zone with blurred PEG spherulites most likely as consequence of high concentration of $C A B$ and certain interactions between both substances, ii) More common PEG spherulite typology most likely with less or no content of $\mathrm{CAB}$ over the effect of the particle surface increment and reduction of inter particle distances. Since the particles packing is increased, the confinement of the organics is increased reducing the effective volumes that will allow a free growth of crystalline lamellas. Comparing both powder types, there is a significant crystallisation temperature reduction in case of INV2 feedstock while these vari ations are less obvious but also occurs with the melting points.

\subsection{Thermal stability studies}

Fig. 7a shows typical simultaneous thermal analysis curves for Invar 36 feedstocks; in this case curves for an INV2 feedstock with 60 vol.\% and a heating rate of $10^{\circ} \mathrm{C} / \mathrm{min}$ are plotted. The normalised DSC curve can be plotted simultaneously with the TGA curve and its derivative, the DTG curve (or the weight loss ratio). Fig. 7b focuses on a comparative of the TGA curves between INV2 feedstocks with different solid fractions. Similar tendencies are observed for INV1 feedstocks.

Table 5 collects some decomposition parameters at $10^{\circ} \mathrm{C} / \mathrm{min}$ of the different feedstock compositions and Table 6 does for the binder and the binder's components. The initial decomposition temperature (IDT) represents the temperature at which the 2 mass $\%$ of the total binder mass was eliminated. The $\alpha_{\max }$ and the $T_{\max }$ represent respectively the conversion and temperature at the maximum weight loss ratio point. The $T_{\max }^{0}$ represents the extrapolated temperature at the maximum weight loss ratio considering a $0{ }^{\circ} \mathrm{C} / \mathrm{min}$ heating rate. The Res refers to the organic residual mass at $600{ }^{\circ} \mathrm{C}$ pondered to the total binder weight and the IPDT to the integral procedure decomposition temperature.

The feedstock organic components decomposition in nitrogen atmosphere occurs in three consecutive overlapped steps as it is deduced from feedstocks DTG curves (Fig. 7a and Fig. 5a-b). The highest decomposition rates occur at high temperatures and correspond to the volatilisations of compounds resulting from the

\section{Table 4}

Characteristic events during the heating and cooling processes of the different feedstocks. $T_{\text {mPEG }}$ : PEG melting temperature; $T_{\text {CPEG }}$ : PEG crystallisation temperature; $\Delta H_{\mathrm{mPEG}}$ : specific enthalpy of fusion relative to the PEG mass; $\Delta H_{\mathrm{cPEG}}$ : specific enthalpy of crystallisation relative to the PEG mass.

\begin{tabular}{|c|c|c|c|c|c|c|c|c|}
\hline \multirow[t]{2}{*}{$\Phi$ [vol.\%] } & \multicolumn{4}{|l|}{ INV1 } & \multicolumn{4}{|l|}{ INV2 } \\
\hline & $\begin{array}{l}T_{\mathrm{mPEG}} \\
{\left[{ }^{\circ} \mathrm{C}\right]}\end{array}$ & $\begin{array}{l}\Delta H_{\mathrm{mPEG}} \\
{\left[\mathrm{J} / \mathrm{g}_{\mathrm{PEG}}\right]}\end{array}$ & $\begin{array}{l}T_{\mathrm{CPEG}} \\
{\left[{ }^{\circ} \mathrm{C}\right]}\end{array}$ & $\begin{array}{l}\Delta H_{\mathrm{CPEG}} \\
{\left[\mathrm{J} / \mathrm{g}_{\mathrm{PEG}}\right]}\end{array}$ & $\begin{array}{l}T_{\mathrm{mPEG}} \\
{\left[{ }^{\circ} \mathrm{C}\right]}\end{array}$ & $\begin{array}{l}\Delta H_{\mathrm{mPEG}} \\
{\left[\mathrm{J} / \mathrm{g}_{\mathrm{PEG}}\right]}\end{array}$ & $\begin{array}{l}T_{\mathrm{CPEG}} \\
{\left[{ }^{\circ} \mathrm{C}\right]}\end{array}$ & $\begin{array}{l}\Delta H_{\mathrm{CPEG}} \\
{\left[\mathrm{J} / \mathrm{g}_{\mathrm{PEG}}\right]}\end{array}$ \\
\hline 57.5 & & & & & 60.9 & 165.8 & 35.5 & 159.2 \\
\hline 60 & 59.1 & 148.9 & 38.4 & 143.8 & 60.6 & 153.0 & 35.9 & 151.3 \\
\hline 62.5 & 59.6 & 146.2 & 39.6 & 142.5 & 59.9 & 155.3 & 33.2 & 147.0 \\
\hline 65 & 59.0 & 150.1 & 39.0 & 145.3 & 56.8 & 154.0 & 31.0 & 147.0 \\
\hline 67.5 & 58.7 & 150.7 & 38.3 & 149.8 & & & & \\
\hline 70 & 57.5 & 128.9 & 35.3 & 128.3 & & & & \\
\hline
\end{tabular}



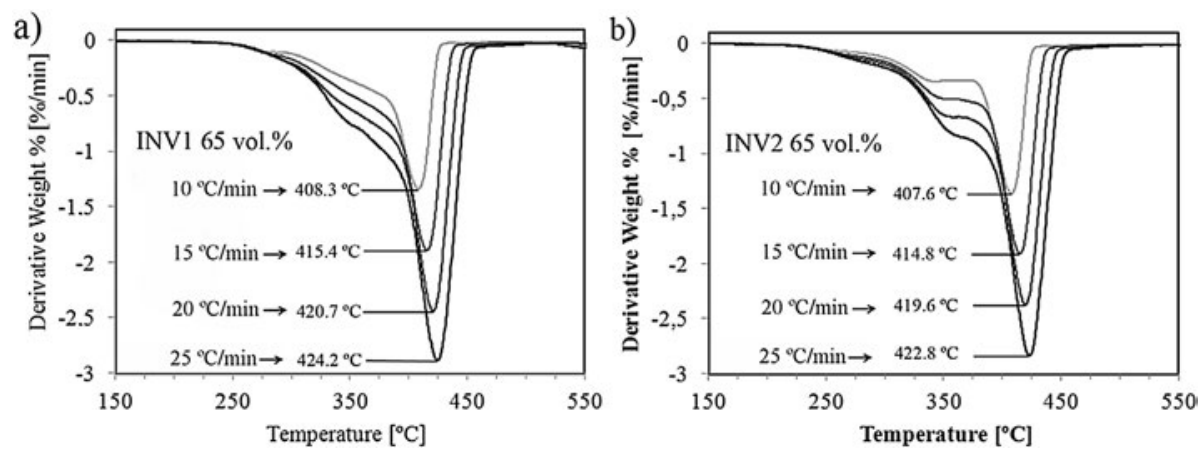

Fig. 5. DTG curves at different heating rates for a) INV1 65 vol.\% feedstock and b) INV2 65 vol.\% feedstock.

degradation of PEG which is the principal component of the binder. The two previous decomposition steps could be linked with the decomposition of CAB. The feedstocks' decomposition behaviour contrasts with a two step binder decomposition process as could be seen in the DTG curves for the single components and the binder system in Fig. 6. The first step occurring in the feedstocks' DTG curves is not registered in the decomposition of the binder system (Fig. 6). The idea that the first decomposition step occurring at low temperatures is associated with an interactive or catalytic effect of the powders over the polymeric compounds is not discarded.

The PEG seems to have a stabilising effect in the decomposition of the $C A B$ as deduced from the displaced DTG peak associated with the $C A B$ in the binder system to higher temperatures. This effect could be revealing some interactions between the CAB and the PEG which is in consonance with DSC results. Furthermore CABs and PEGs decomposition peaks tend to overlap in the feedstocks DTG curves. Although certain compatibility is assumed between CABs and PEGs, their miscibility is not complete as it was contrasted with well differentiated $C A B$ rich and PEG rich regions in the binder system (Fig. 4). The kneading effect that the powder particles exerts over the binder components may cause a smaller and closer PEG rich and $C A B$ rich regions or increase the miscibility between the molecules in case there is any. The CAB and PEG polymer chains may be more effectively entangled and the formation of weak links could be promoted. This would explain the displacement and overlapping of these substances decomposition peaks, presumably because of a hindering of the elimination of the CABs' decompo sition components entrapped in these smaller domains.

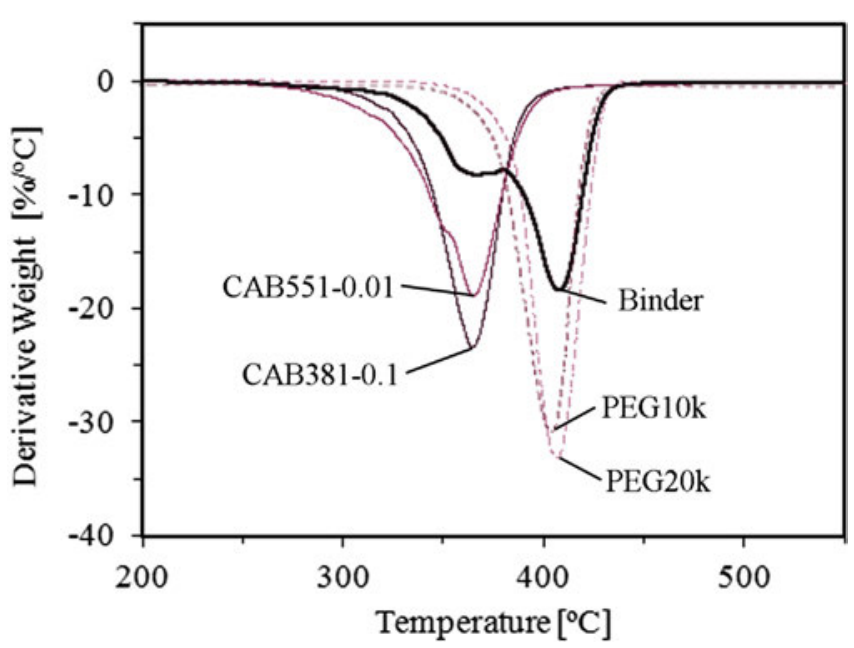

Fig. 6. Comparative of DTG curves of the binder system and the binder components.
The IDT of the binder system coincides with the minimum IDT of its components that corresponds to CAB551. However, there is a considerable reduction of the IDT values registered in the feed stocks and there is a clear dependence on the powder type and the solid loading. The lowest IDT values correspond to the INV2 feed stocks with high solid loadings. There is a direct relation of the total powder surface area with the IDT value. The main idea is that the mixing process favours the creation of weak links between poly mers or between polymers and particles. These weak links are preferential points for the polymer chain scission during thermal degradation. It is well established that PEG thermal degradation proceeds by the random chain scission of $\mathrm{C}-\mathrm{O}$ bonds [25]. Ther mally promoted hydrogen abstraction reactions of hydroxyl radi cals with $\mathrm{C}-\mathrm{O}$ is one possible mechanism of chain scission. This reaction is kinetically more favourable than the own homolytic cleavage of $\mathrm{C}-\mathrm{O}$ [26]. As the solid fraction increases or the powder particle size is reduced, there is more available surface area for the creation of hydrogen bond or weak links favouring a subsequent catalytic effect at higher temperatures.

The CABs do not completely volatilise at $600{ }^{\circ} \mathrm{C}$ in nitrogen at mosphere and leave a solid organic residue. The proportion of this organic residue with respect to the total binder is reduced in INV1 feedstocks in comparison with the INV2 feedstocks and the sole binder, both having similar values. As the solid loading increases, the residue increases. Although the powder surface may be having a catalytic effect on the polymers decomposition, it could be also a site for the formation of a thermally stable char layer. As the powder surface increases there are more sites for a char layer formation. There is also the effect of the powder particle packing increment with the solid loading which complicates the evacuation of volatiles. In Fig. $7 \mathrm{~b}$ a reduction of the weight loss rate and the displacement of the TGA curve to higher temperatures as the solid loadings increases is observed. This behaviour is in consonance with the possible hin dering effect of the char layer formation around particles and the reduction of the evacuation pore net channels sizes.

The variation of the IPDT values with the solid loading for INV1 and INV2 is shown in Fig. 8. The IPDT increases with solid loading which is predictable by the variation of the $S_{2}$ area because of the increment of the metallic part. The typical linear tendency is observed, but the slope and the IPDT values for the same solid loading vary with the heating rates and particle type. A change in the slope takes place at 62.5 vol.\% and 67.5 vol.\% for the INV2 and INV1 respectively. This change in the slope coincides with the values of optimal solid loading around these compositions that were determined using several different methodologies in previous investigations with Invar 36 feedstocks. These results also support previous works that proposed this methodology as an alternative or a complement to conventional methodologies for assessing optimal solid loadings in ceramic powders [8]. 

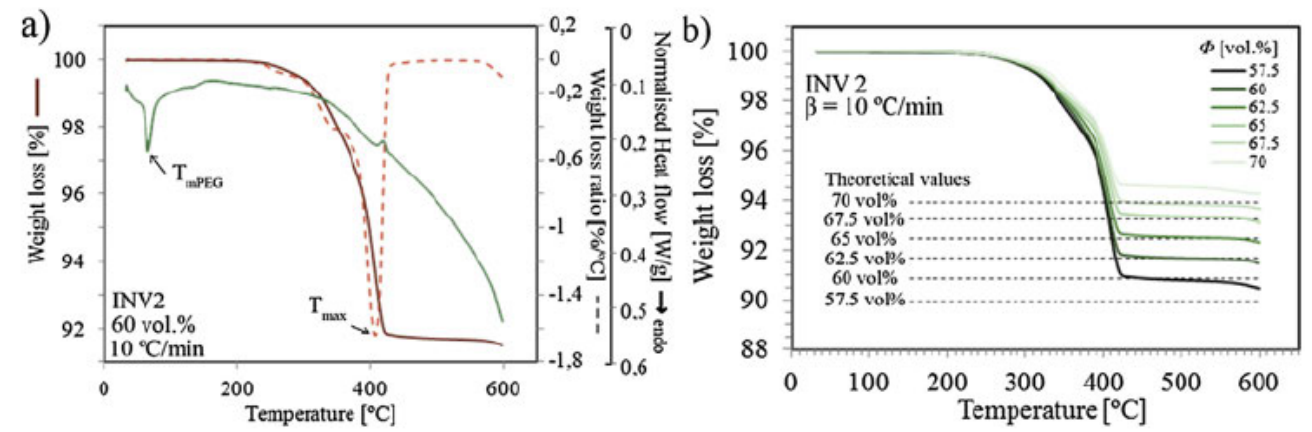

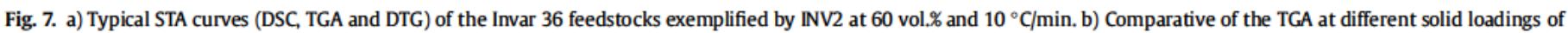
INV2 feedstocks as $10^{\circ} \mathrm{C} / \mathrm{min}$.

Table 5

Comparative of different degradation parameter for different INV1 and INV2 feedstock at different solid loadings.

\begin{tabular}{|c|c|c|c|c|c|c|c|c|c|c|c|c|}
\hline \multirow[t]{2}{*}{$\Phi[\mathrm{vol} . \%]$} & \multicolumn{6}{|l|}{ INV1 } & \multicolumn{6}{|l|}{ INV2 } \\
\hline & IDT $\left[{ }^{\circ} \mathrm{C}\right]$ & $\alpha_{\max }[\%]$ & $T_{\max }\left[{ }^{\circ} \mathrm{C}\right]$ & $T_{\max }^{0}\left[{ }^{\circ} \mathrm{C}\right]$ & Res [\%] & IPDT $\left[{ }^{\circ} \mathrm{C}\right]$ & IDT $\left[{ }^{\circ} \mathrm{C}\right]$ & $\alpha_{\max }[\%]$ & $T_{\max }\left[{ }^{\circ} \mathrm{C}\right]$ & $T_{\max }^{0}\left[{ }^{\circ} \mathrm{C}\right]$ & Res [\%] & IPDT $\left[{ }^{\circ} \mathrm{C}\right]$ \\
\hline 57.5 & & & & & & & 261.2 & 72.5 & 407.5 & 276.7 & 5.4 & 8714 \\
\hline 60 & 265.7 & 73.0 & 408.1 & 278.1 & 3.0 & 9260 & 259.9 & 75.5 & 407.2 & 285.2 & 5.7 & 9839 \\
\hline 62.5 & 261.4 & 74.2 & 408.3 & 279.6 & 3.4 & 10,388 & 257.8 & 75.8 & 407.1 & 290.2 & 6.4 & 10,955 \\
\hline 65 & 259.3 & 75.4 & 408.3 & 280.3 & 3.7 & 11,513 & 256.3 & 75.6 & 407.8 & 285.8 & 6.8 & 12,263 \\
\hline 67.5 & 257.9 & 74.5 & 407.8 & 281.3 & 3.5 & 12,655 & 248.1 & 75.7 & 407.7 & 284.1 & 6.3 & 13,527 \\
\hline 70 & 243.4 & 75.6 & 407.7 & 284.3 & 4.7 & 14,776 & 241.1 & 75.5 & 407.1 & 282.3 & 7.0 & 14,774 \\
\hline 72.5 & 246.0 & 75.3 & 407.2 & 273.2 & 4.6 & 16,689 & & & & & & \\
\hline
\end{tabular}

This change in the slope may be explained assuming a char layer formation around the powder particles. The optimal solid loading is very near to the CPVC with the difference that in the first situation there is still a thin coating of polymers embedding the particles. This polymer coating enlarges as the solid loading is reduced. For low solid fractions, despite a char layer formation is most likely taking place, there could be still enough separation between par ticles to allow an effective removing of all the decomposition vol atiles out of the green body. Nevertheless at optimal solid loading compositions the char layers of neighbouring particles would collide hindering the volatiles evacuation causing the slope change. This slope change is telling that for subsequent solid loading in crements the variation of the $S_{2}$ areas is approximately constant while the values of the $S_{1}$ areas varies more abruptly (due to a reduction of the weight loss ratio and a displacement of the TGA curves to higher temperatures).

\subsection{Degradation kinetics}

The KAS and FWO methods allow determining the activation energy at different conversion values. Fig. 9 shows the dependence on the conversion of the apparent activation energy values, as assessed by the KAS method of the main binder components and the binder system while Fig. 10 does it for the INV1 and INV2 feedstocks at different solid volumetric fractions. Similarities are found in the curves using the FWO method thus this graph was

Table 6

Comparative of different degradation parameter for binder system and binder components. The maximum degradation rate temperature is $404.5^{\circ} \mathrm{C}$ for the binder, but the peak corresponding to CABs' degradation is also presented.

\begin{tabular}{lcccrrr}
\hline & PEG10k & PEG20k & CAB381 & CAB551 & Binder & \\
\hline IDT [ ${ }^{\circ} \mathrm{C}$ ] & 351.4 & 366.3 & 301.4 & 285.7 & 283.9 & \\
IPDT [ $\left.{ }^{\circ} \mathrm{C}\right]$ & 412.5 & 413.3 & 442.9 & 447.1 & 432.2 & \\
Res [\%] & 0.1 & 0.1 & 7.9 & 8.6 & 6.4 & \\
$T_{\max }\left[{ }^{\circ} \mathrm{C}\right]$ & 401.2 & 406.8 & 364.6 & 363.6 & 366.7 & 404.5 \\
\hline
\end{tabular}

obviated. The KAS method uses a more accurate approximation of the integral temperature than FWO and it is considered to produce more reliable results of the activation energy [21].

The activation energies for the PEG20k maintain approximately constant with $\alpha$ variations, which likely indicates a single rate limiting decomposition step over the entire reaction duration. The averaged value of $E_{\mathrm{a}}$ using FWO method was $148 \mathrm{~kJ} / \mathrm{mol}$ and in case of KAS method $144 \mathrm{~kJ} / \mathrm{mol}$. They properly suit with the typical PEGs activation energies interval $(120-200 \mathrm{~kJ} / \mathrm{mol})$ reported in literature [25]. In the case of CAB there is a slight increment of the activation energy with $\alpha$ from $57 \mathrm{~kJ} / \mathrm{mol}$ for $\alpha \quad 0.2-97 \mathrm{~kJ} / \mathrm{mol}$ for $\alpha \quad 0.8$ as determined with FWO and from $50 \mathrm{~kJ} / \mathrm{mol}$ for $\alpha \quad 0.2-91 \mathrm{~kJ} / \mathrm{mol}$ for

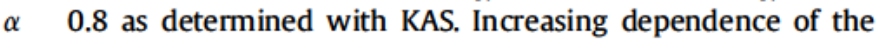
activation energy on the conversion is not unusual in polymer degradation [27]. This behaviour is considered to be an indicator of a complex reaction [28]. Vyazovkin and Lesnikovich $[29,30]$ noted

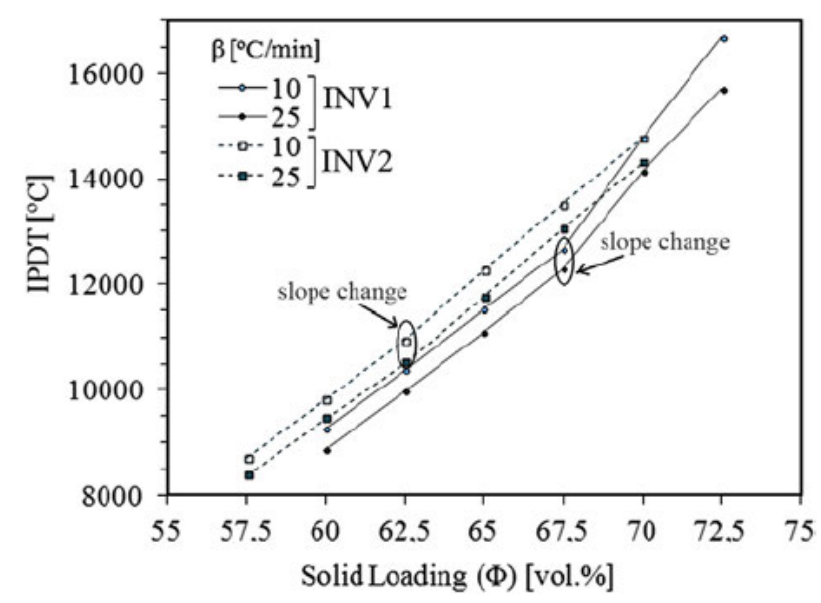

Fig. 8. IPDT values evolution at different solid loadings, heating rates and for INV1 and INV2 feedstocks. 


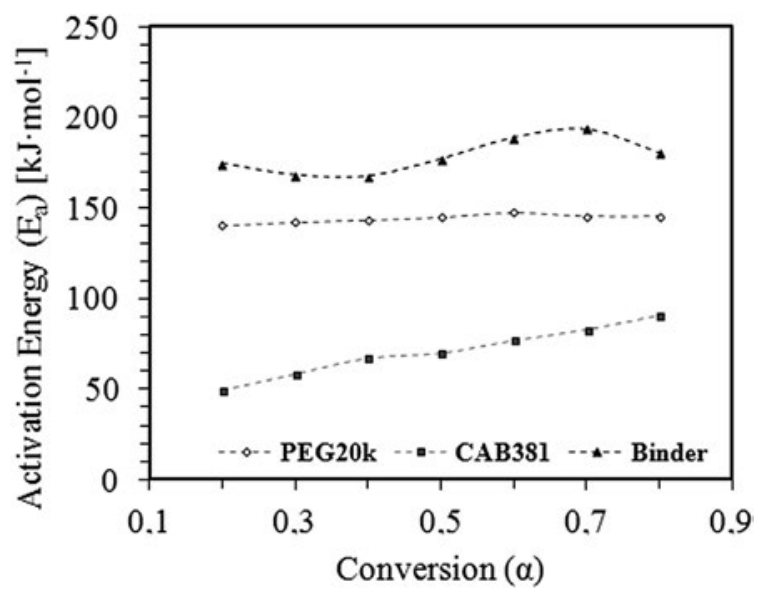

Fig. 9. Evolution of the activation energies with the conversion calculated with the KAS method for the main binder components and for the own binder system.

that in non isothermal experiments, patterns in the shape of the curve dependence of the apparent value of $E_{\mathrm{a}}$ on $\alpha$ could provide insight into the type of complex process taking place. An increasing dependence of $E_{\mathrm{a}}$ on $\alpha$ characterises concurrent, competitive re actions, whereas convexity indicates a change in the rate determining step. In the case of the binder system, the activation energies are higher than those for the single PEG and CAB. Several stages are observed. From conversion 0.2 to 0.4 a stable value of the $E_{\mathrm{a}}$, with an average value of $172 \mathrm{~kJ} / \mathrm{mol}$ calculated with FWO and $170 \mathrm{~kJ} / \mathrm{mol}$ in case of KAS, is observed. Then $E_{\mathrm{a}}$ increase up to $195 \mathrm{~kJ} / \mathrm{mol}$ calculated with FWO and $194 \mathrm{~kJ} / \mathrm{mol}$ in case of KAS for $\alpha \quad 0.7$. These results could agree with the suggested interactions between PEG and CAB observed by DSC. Grafting or cross linking reactions between $C A B$ and $P E G$ functional groups presumably occurs creating more thermally stable reaction nucleus and thus increasing the activation energy. It is not the scope of this work to study the nature of those reactions which would be also very limited by the employed techniques.

Through $\alpha$, the feedstocks seem to have an important influence on $E_{\mathrm{a}}$ that is independent of solid loading. Two stages are mainly observed. For the majority of the curve, $E_{\mathrm{a}}$ increases abruptly as the values of $\alpha$ increase up to a value at which $E_{\mathrm{a}}$ either stabilises or begins to decrease; this behaviour gives the curve a certain con vexity. At low conversion rates the activation energy is lower than the binder's (taking $\alpha \quad 0.3$, Ea is less than $170 \mathrm{~kJ} / \mathrm{mol}$ for almost all the feedstocks employing KAS method) which is suggesting that the degradation at this point is favoured by a catalytic effect of the powders. The concurrent reactions presumed from the $E_{\mathrm{a}}$ incre ment could be molecular degradation reactions of both $C A B$ and
PEG and other reactions that hinder the volatiles evacuation as well as the formation of a thermally stable layer around the particles (by recombination of degraded polymers forming crosslinks or for mation of a char layer). The nature of this thermally stable layer and the reactions involving it are uncertain, but its formation is sup ported by the observation of an organic residue after TGA experi ments. This residue is also observed in the thermal degradation of the studied CABs in nitrogen atmosphere. The subsequent plateau at high conversion values may indicate the end of a reaction or the creation of open pathways that facilitate the removal of volatiles as the binder decomposes. That last would suit with a slight pro gressive decrease of the activation energy at high temperatures. In many of the feedstocks, the value of the Ea in the plateau at high conversion rates is higher than $195 \mathrm{~kJ} / \mathrm{mol}$ (KAS), which is higher activation energy than those for the binder. This evidences that the presence of the powder influences negatively in the volatilisation of the break down molecules at high conversion rates.

Fig. 11 represents the activation energies for a constant con version of 0.5 and for different INV1 and INV2 feedstocks using KAS and FWO methods. In this case a minimum in the activation energy vs. the solid loading curves is observed at 62.5 vol.\% and 70 vol.\% for INV2 and INV1 respectively. It is worth to consider that the apparent activation energy summarises different effects involving the degradation of polymers, but at the end, as measured from TGA, $E_{\mathrm{a}}$ reflects how effective are the resulting break down compounds volatilised. Degradation may begin at temperatures lower than the registered by TGA. However, a weight loss only would be appreci ated when the decomposition species had a certain low molecular weight that allows for the volatilisation or for the mass transport trough different mediums, e.g. melted polymer, evacuating inter particle pathways, etc. In that respect, the occurrence of a mini mum in the Ea vs. solid loading curve may be explained by opposing effects. First, an increment of the weak links as the solid loading increases, i.e. an increment of catalytic decomposition sites, may reduce the apparent degradations activation energy by favouring the fragmentation rate of the polymer chains. The opposite effect, an increase in the apparent activation energy, would occur as a consequence of a better particle packing as the solid loading increases, reducing the diameters of the volatiles removal channels. This effect may have been enhanced by the formation of a thermally stable layer of char around the particles. All of these effects combine to minimise the apparent activation energy of degradation.

Unfortunately non isothermal methods without other Supporting information have generally a no mechanistic value, but at least in this case could serve as a first rough approach to support the general analysis in combination with other methodologies presented. The KAS and FWO methods may introduce errors because they are based on an approximation of the temperature
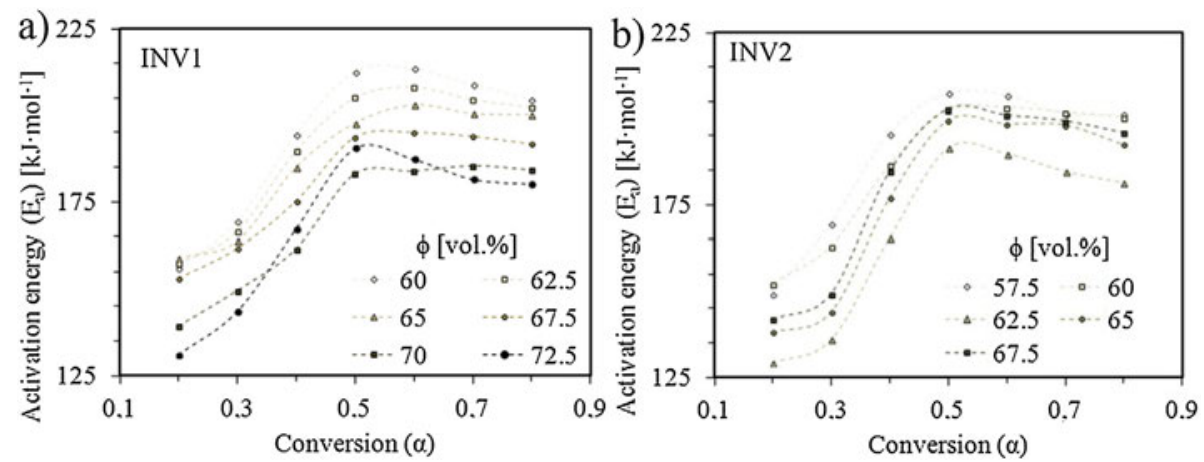

Fig. 10. Evolution of the activation energies with the conversion calculated with the KAS method at different solids loading for a) INV1 feedstocks and b) INV2 feedstocks. 


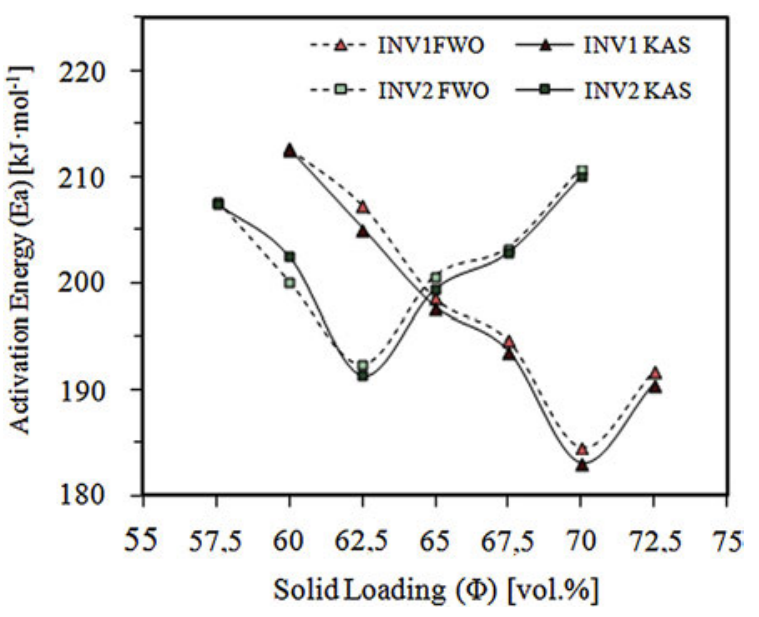

Fig. 11. Activation energies evolution with solid loadings of INV1 and INV2 feedstocks calculated by FWO and KAS methods.

integral $[15,18]$, but in any case it seems that the results are reaf firming 62.5 vol.\% or 67.5 vol.\% as the optimum solid loading value with respect to both rheological concerns and thermal stability studies of the feedstocks. The direct relationship between the apparent activation energies, with respect to both rheological and thermal degradation concerns, is not clear. There are analogies in the possible explanations of the mechanisms that affect these fac tors, such as the change of the particle packing and the effective interaction surface between the organic compounds and the par ticles. Nevertheless, studying the thermal degradation by calcu lating activation energies for different solid loading conditions emerges as an alternative to the traditional rheological studies used to assess optimal solid loading in PIM feedstocks.

\section{Conclusions}

Thermal analyses via DSC and TG experiments in a nitrogen atmosphere were performed for different solid loading Invar 36 feedstocks composed of CAB and PEG. Two different powders sizes were selected. Binder components in presence of powder particles apparently thermally degrade by concurrent reactions in three steps between $250^{\circ} \mathrm{C}$ and $450{ }^{\circ} \mathrm{C}$. A possible interaction between the Invar 36 particles and the binder components was revealed by DSC analysis. Thermal stability studies using the IPDT factor reveal a linear slope in the feedstock thermal stability as the solid loading increases. A change in the linear slope occurs at approximately 62.5 vol.\% solid loading for fine powder whereas this change occurs at 67.5 vol.\% for the coarse powders. This change coincides with the optimal solid loading calculated in previous studies using rheo logical methods. The kinetics of the thermal degradation of the binder components in terms of calculated apparent activations energies were also studied using the FWO and KAS integral methods. Variations in the apparent activation energies calculated using these methods suggest the occurrence of complex, concur rent and competitive reactions. A representation of the apparent activation energy at a constant conversion for different solid loadings shows that the activation energy is minimised at $62.5 \mathrm{vol} . \%$ and 70 vol.\% for fine and coarse powders respectively. This value coincides with a minimum in the activation energy calculated using rheological methods in previous studies. The possible effects that lead on this minimum were discussed. It seems that the powder has a catalytic effect upon the binder decomposition but at the same time particles may be hindering the evacuation of volatiles. All of these results emphasise the potential for using thermocalorimetric analysis instead of, or as a complement to, conventional rheological studies to determine the optimal solid loading of MIM feedstocks.

\section{Acknowledgements}

The authors a wish to thank GUZMÁN GLOBAL S.L. and MIM TECH ALFA for their collaboration on the ECOPIM project (ref. IPT 2011093120000 ) that was funded by the Spanish Ministry of the Economy and Competitiveness. Furthermore, the authors would like to acknowledge the strong support from the ESTRUMAT projects (ref. S2009/MAT 1585), which were funded by the CAM Consejería Educación Dir. Gral. Universidades e Investigación, and from the COMETAS project (ref. MAT2009/14448 C02 02), which was funded by the Spanish Ministry of the Economy and Compet itiveness. J.P.F. B. acknowledges support from "Marie Curie" Amarout Europe Program.

\section{References}

[1] Petzoldt F. Current status and future perspectives of the MIM technology. CFICeram Forum Int 2012;89:E11 5.

[2] German R. Metal powder injection molding (MIM): key trends and markets. In: Heaney D, editor. Handbook of metal injection molding. Woodhead Publishing Limited; 2012.

[3] Yun JW, Lombardo SJ, Krueger DS, Scheuer P. Effect of decomposition kinetics and failure criteria on binder-removal cycles from three-dimensional porous green bodies. J Am Ceram Soc 2006;89:176 83.

[4] Liau LCK, Chiu CC. Optimal heating strategies of polymer binder burnout process using dynamic optimization scheme. Ind Eng Chem Res 2005;44: 458693.

[5] Aggarwal G, Park S-J, Smid I, German RM. Master decomposition curve for binders used in powder injection molding. Metall Mater Trans A Phys Metall Mater Sci 2007;38A:606 14.

[6] Belgacem M, Thierry B, Jean-Claude G. Investigations on thermal debinding process for fine 316L stainless steel feedstocks and identification of kinetic parameters from coupling experiments and finite element simulations. Powder Technol 2013;235:192 202.

[7] Salehi M, Clemens F, Graule T, Grobety B. Kinetic analysis of the polymer burnout in ceramic thermoplastic processing of the YSZ thin electrolyte structures using model free method. Appl Energy 2012;95:147 55.

[8] Hidalgo J, Jiménez-Morales A. Torralba JM. Thermal stability and degradation kinetics of feedstocks for powder injection moulding a new way to determine optimal solid loading? Polym Degrad Stab 2013;98:1188 95.

[9] Jin F-L, Park S-J. Thermal properties of epoxy resin/filler hybrid composites. Polym Degrad Stab 2012;97:2148 53.

[10] Doyle CD. Estimating thermal stability of experimental polymers by empirical thermogravimetric analysis. Anal Chem 1961;33:77.

[11] Vyazovkin S. Model-free kinetics staying free of multiplying entities without necessity. J Therm Anal Calorim 2006;83:45 51.

[12] Holland BJ, Hay JN. The value and limitations of non-isothermal kinetics in the study of polymer degradation. Thermochimica Acta 2002;388:253 73.

[13] Flynn JH, Wall LA. A quick direct method for determination of activation energy from thermogravimetric data. J Polym Sci Part B Polym Lett 1966;4:323.

[14] Ozawa T. A new method of analyzing thermogravimetric data. Bull Chem Soc Jpn 1965;38:1881.

[15] Doyle CD. Series approximations to equation of thermogravimetric data. Nature 1965;207:290.

[16] Kissinger HE. Reaction kinetics in differential thermal analysis. Anal Chem 1957;29:1702 6.

[17] Akahira T, Sunose T. Joint convention of four electrical institutes. Chiba: Chiba Institute of Technology; 1971. p. 2231.

[18] Coats AW, Redfern JP. Kinetic parameters from thermogravimetric data. Nature 1964;201:68.

[19] Bernardo E, Hidalgo J, Jimenez-Morales A, Torralba JM. Feedstock development for powder injection moulding of zirconium silicate. Powder Inject Mould Int 2012;6:4.

[20] Minseok S, Min Soo P, Jin Kon K, Il Bum C, Kyung Ho K, Hwan Jin S, et al. Water-soluble binder with high flexural modulus for powder injection molding. J Mater Sci 2005;40:11059.

[21] Vyazovkin S, Burnham AK, Criado JM, Perez-Maqueda LA, Popescu C, Sbirrazzuoli N. ICTAC Kinetics Committee recommendations for performing 
kinetic computations on thermal analysis data. Thermochimica Acta 2011;520:1 19.

[22] Tanaka Y, Saito H, Tsutsumi Y, Doi H, Imai H, Hanawa T. Active hydroxyl groups on surface oxide film of titanium, 316L stainless steel, and cobaltchromium-molybdenum alloy and its effect on the immobilization of poly(ethylene glycol). Mater Trans 2008;49:805 11.

[23] Derosa RL, Trapasso JA. Poly(ethylene glycol) interactions with alumina and silica powders determined via DRIFT. J Mater Sci 2002;37:1079 82.

[24] Rubio J, Kitchener JA. Mechanism of adsorption of poly(ethylene oxide) flocculant on silica. J Colloid Interface Sci 1976;57:132 42.

[25] de Sainte Claire P. Degradation of PEO in the solid state: a theoretical kinetic model. Macromolecules 2009;42:3469 82
[26] Vaghjiani GL, Ravishankara AR. Kinetics and mechanism of hydroxyl radical reaction with methyl hydroperoxide. J Phys Chem 1989;93:12.

[27] Vyazovkin S, Sbirrazzuoli N, Dranca L. Variation in activation energy of the glass transition for polymers of different dynamic fragility. Macromol Chem Phys 2006;207:1126 30.

[28] Elder JP. Multiple reaction scheme modeling 3: mutually independent nth order reactions. J Therm Anal 1989;35:1965 84.

[29] Vyazovkin SV, Lesnikovich AI. On the dependence of kinetic parameters and functions in non-isothermal kinetics. Thermochimica Acta 1987:122:413 8.

[30] Vyazovkin SV, Lesnikovich AI. An approach to the solution of the inverse kinetic problem in the case of complex processes.1. Methods employing a series of thermoanalytical curves. Thermochimica Acta 1990;165:273 80. 